\title{
سرير التحنيط في الفن المصري القديم
}

هدى محمد عبد المقصود

أستاذ الأثار والحضارة المصرية القديمة

قسم الآثار - كلية الآداب - جامعة المنيا

\section{ملخص البحث: :}

يعتبر منظر سربر التحنيط من المناظر التي شاع ظهورها منذ بداية الدولة الحديثة على كثير من الآثار المصرية القديمة ، يظهر المنظر دائما بعناصر شبه ثابتة ، تتكون من مومياء المتوفي راقداً على سربر التحنيط سواء كان في مراحل التحنيط المختلفة أو في صورة المومياء المختلفة .

ودائماً يقف إلى جانب السرير المعبود أنوبيس ، وعند رأسه أقدام السرير تقف المعبودنان • إيزبس ونفتيس

وقد اهتم البحث باتجاه تصوير المومياء وسرير التحنيط والتي اتضح من الدراسة أنهما غالباً يتجهان ناحية اليسار عندما تتتهي عملية التحنيط ، كما يستخدم في مراحل التحنيط الأولى سرير مكون من مجرد ألواح خثبية أما في صورتها النهائية فهو يوضع على سرير يأخذ عادة رأس

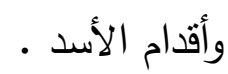

\section{الكمات الدالة}

$$
\text { - إيزبس }
$$$$
\text { 1- أنوبيس }
$$

$$
\text { ؟- عملية التحنيط }
$$

$$
\text { م- سرير التحنيط }
$$

\section{سرير التحنيط في الفن المصري القديم}

يعتبر منظر سرير التحنيط من المناظر منكررة الظهور في الفن المصري القيم ، حيث يظهر ضدن زخارف جدران التوابيت وعلى الأكفان وفي نقوش المقابر وعلى بعض التماثيل وفي نقوش البرديات'

يرجع أقدم ظهور لهذا المنظر الي عصر الدولة الحديثة، واستمر ظهوره حتي العصر

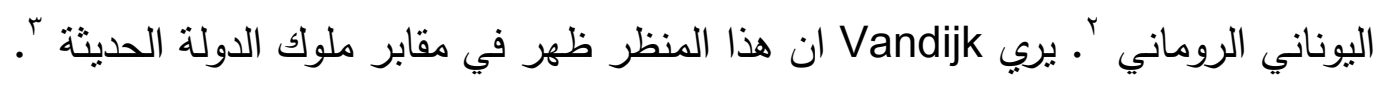




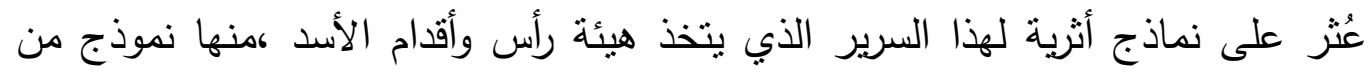

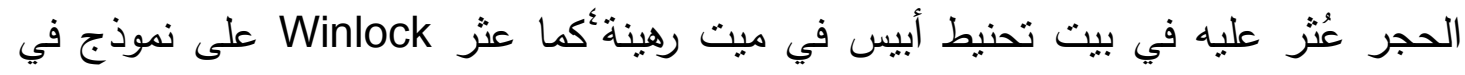
الدير البحري ـ

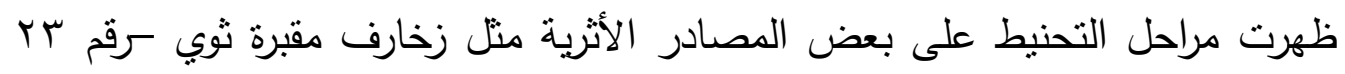

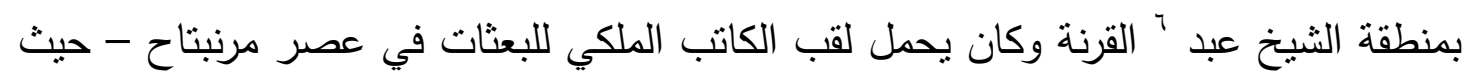

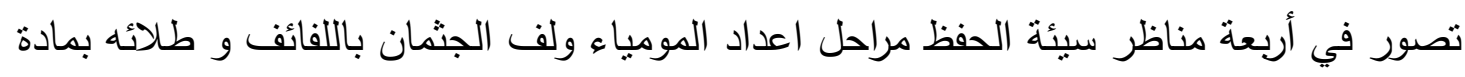

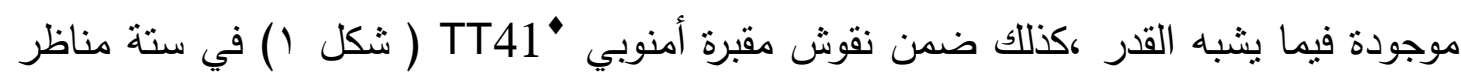

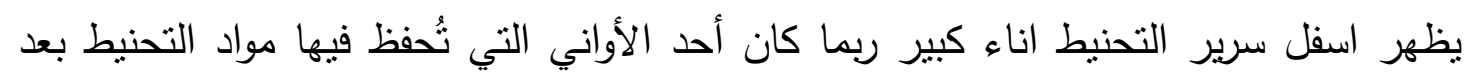

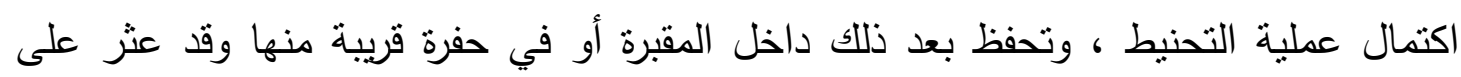

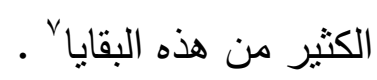

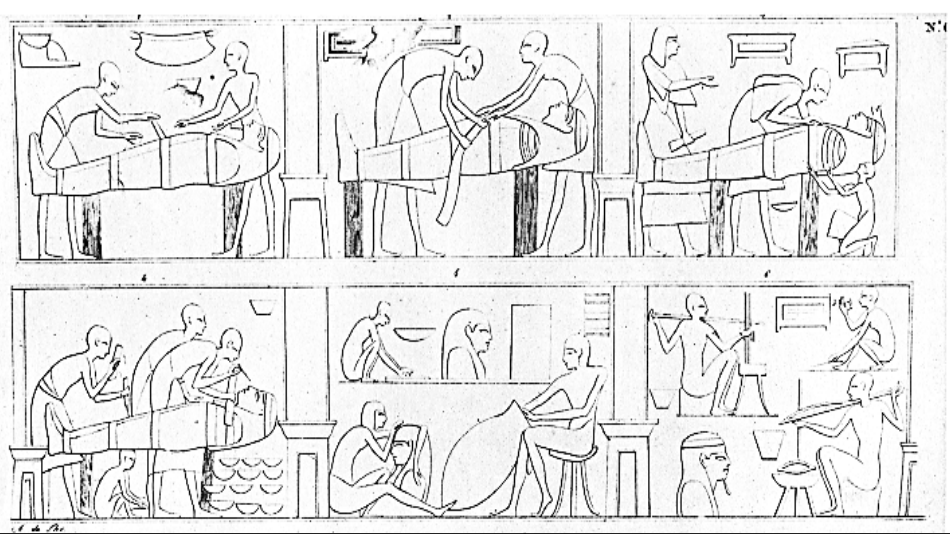

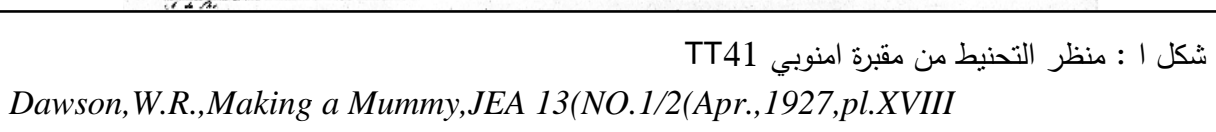

كذلك ضمن نقوش مقبرة سننجم بدير الدينة، ، حيث نجد في مراحل إعداد المومياء سرير التحنيط في اتجاه اليمين ، بينما عندما اكتمل اعدادها اتجه سرير التحنيط ورأس المومياء جهية التهاء

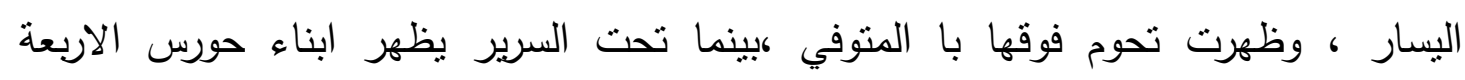

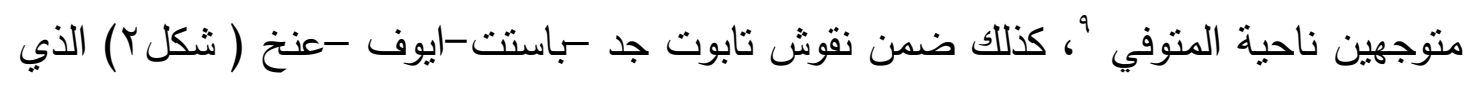

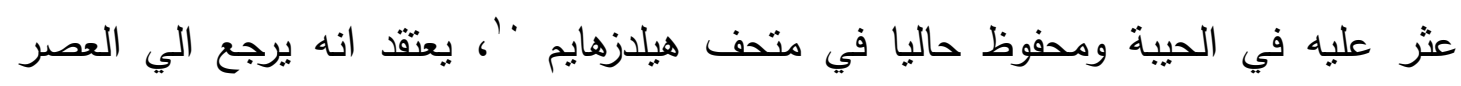

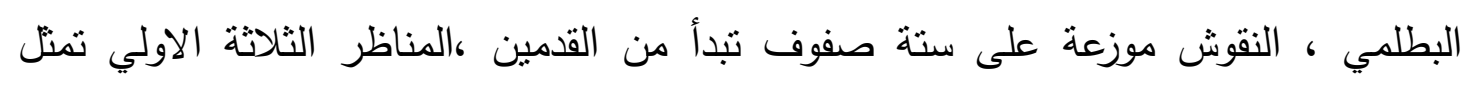

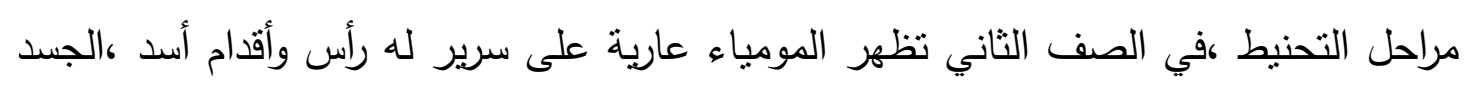


مرفوع عند الرأس والأقدام على مساند بينما تتبت حبوب القمح أسفل المومياء -أثنارة الي ارتباط

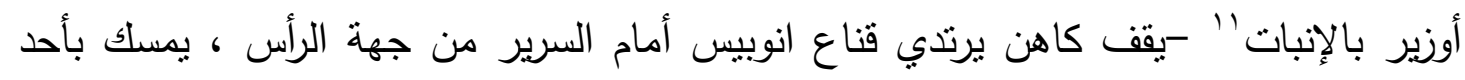

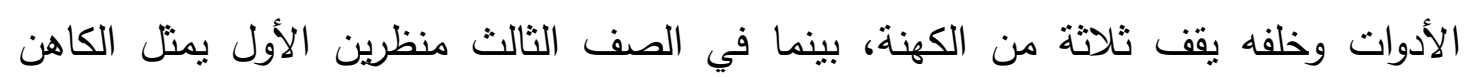

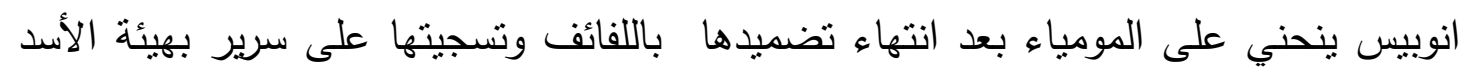

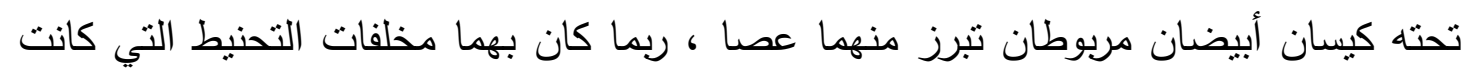

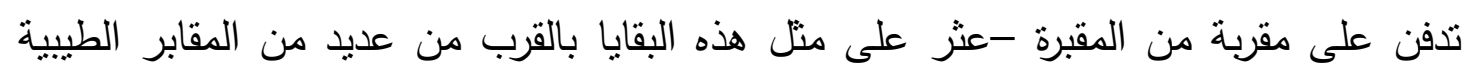

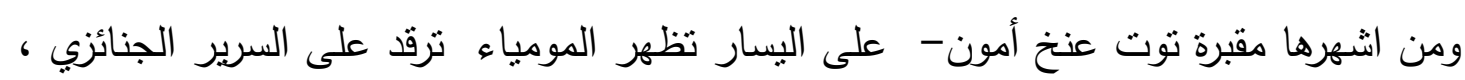

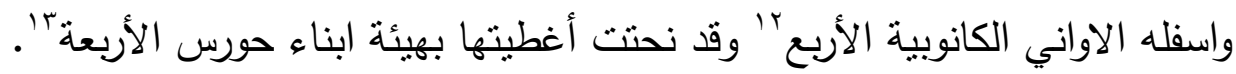

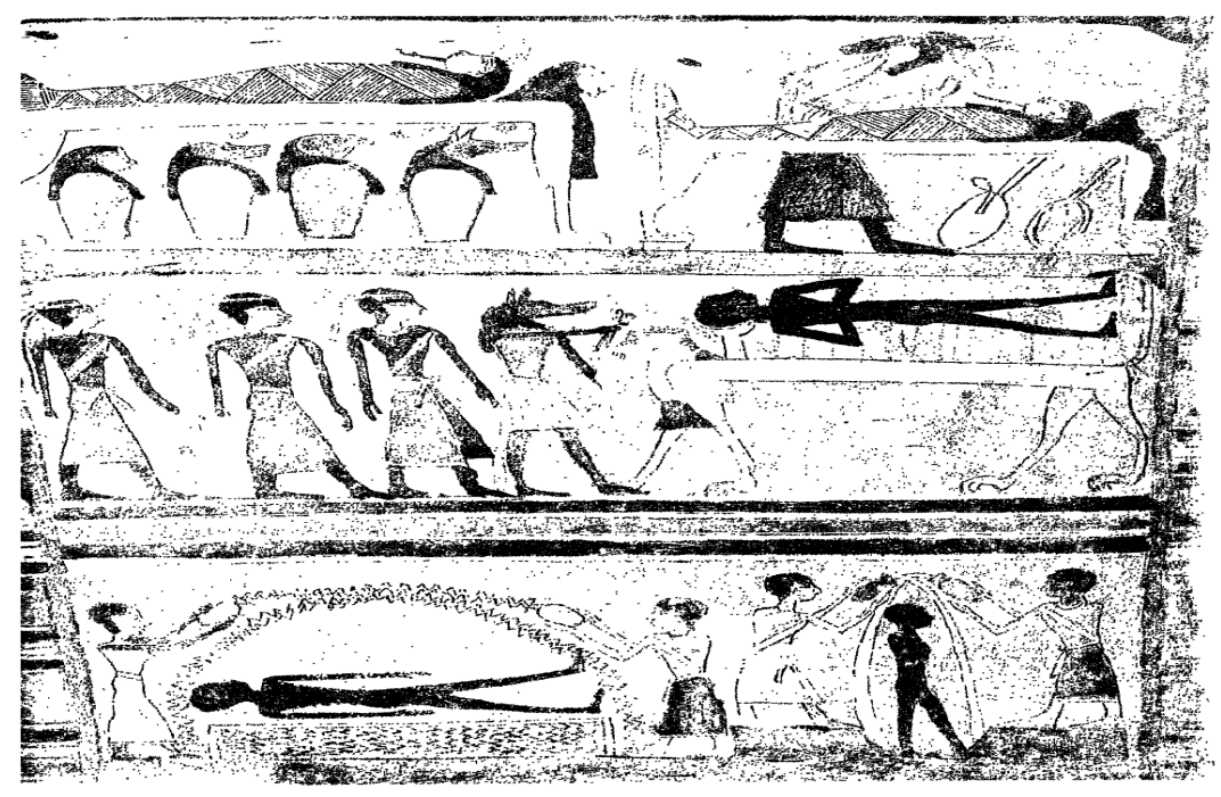

inv.no.1954 شكل r : نقوش تابوت جد باستت /يوف عنخ - متحف هلدزهايم Schmitz,B.,Sarg des Djed-Bast-iu-ef-ankh,in:A.Eggebrecht,ed., Stuche nach Unsterblichkeit Hildesheim and Mainz,1990,p.28.

يُلاحظ في المناظر السابقة أن في مراحل تطهير المومياء واعدادها يكون اتجاه سرير التحنيط ورأس المومياء جهة اليمين بينما بعد تضميدها باللفائف يكون اتجاه السرير ورأس التهاء

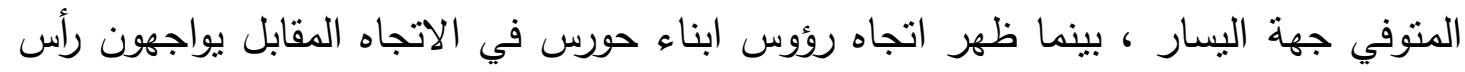
المتوفي. 
يظهر منظر سرير التحنيط كذلك مصاحبا للفصل 101 من كتاب الموتى (شكل )؛ يفسر هذا المنظر عادة انه يمنل غرفة الدفن مزودة بالصيغ المناسبة لكل الطقوس المرتبطة بالتحنيط والإحياء والجنازة .

كما صُور على ظهر تمثال كي نبو - محفوظ بمتحف هنوفر - Hannover,Kestner نقش يمثل صفان من المناظر يعلوهما علامة السماء ، الصف العلوي 2945 يتوسطه انوبيس ينحني على مومياء أوزير الراقد على سرير التحنيط الذي أخذ هيئة الأسد ،

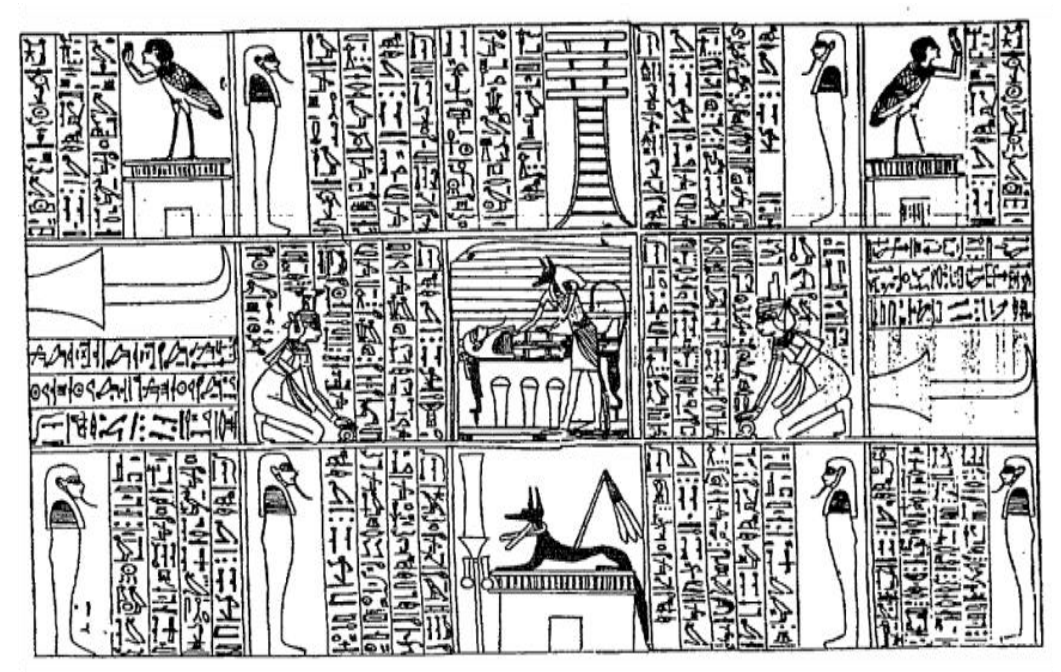

النص امام انوبيس يقول inbw imy wt nb Dsrw " انوبيس الذي فوق خبيته سبي الجبانة "،بينما كتب فوق اوزير wsir xnty imnty wnn nfr nb Dt " أوزبر الذي في مقدمة الغربيين وننفر سبي الأبدية" ،اسفل السرير تظهر مقصورة صغيرة يربض عليها انوبيس ،امام السرير تقف المعبودة ايزيس وقد كتب فوقها : ist wrt mwt nTr snt nTr aA nb imnt العظبم سبي الأبدية" ، وعلى الجانب الأخر تقف نبت حت مواجهة لأيزيس "ال.

يلفت Vandijk النظر الي بعض مناظر سرير التحنيط على سبيل المثال في غرفة دفن شاشانق الثالث في تانيس نقش بمثل سربر تحنيط يميز هذا المنظر ان المومياء ترفع رأسها في اثثارة الي البعث الذي ثم بواسطة حور الذي يقترب من المومياء موجها الي أنفه صولجان

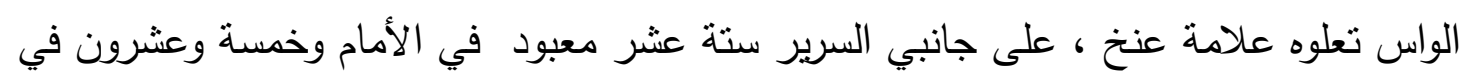
الخلف ينتظرون الحدث ل'، قام W.Waitkus و بتحليل المنظر وبمقارنته بمناظر

ז. 
مشابهة في كلا من الأوزيرون بأبيدوس وغرفة دفن رمسيس الثالث(شكلء) ومقبرة أمنرديس

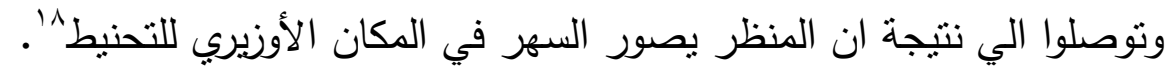

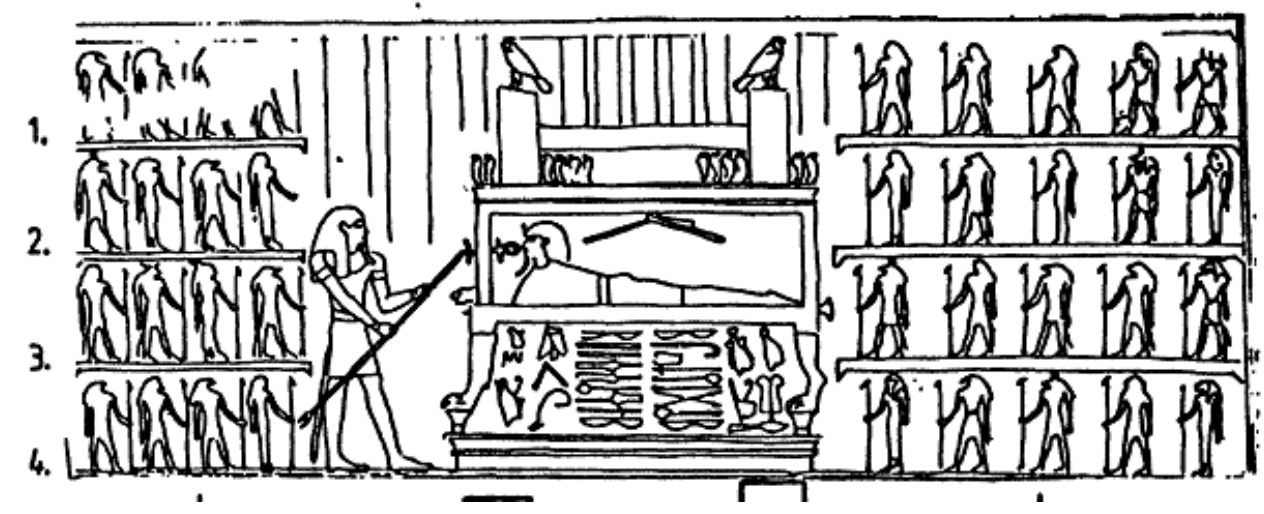

$$
\begin{aligned}
& \text { شكل ع : سرير التحنيط بغرفة دفن رمسيس الثالث ، نقلاً عن : } \\
& \text { Wiatkus,W.,op.cit.,abb.2,p.70 }
\end{aligned}
$$

أما في منظر التحنيط في مقبرة خع بخنت TT2 • (ثكله) فقد استبدل جسد أُوزير بسمكة AbDw

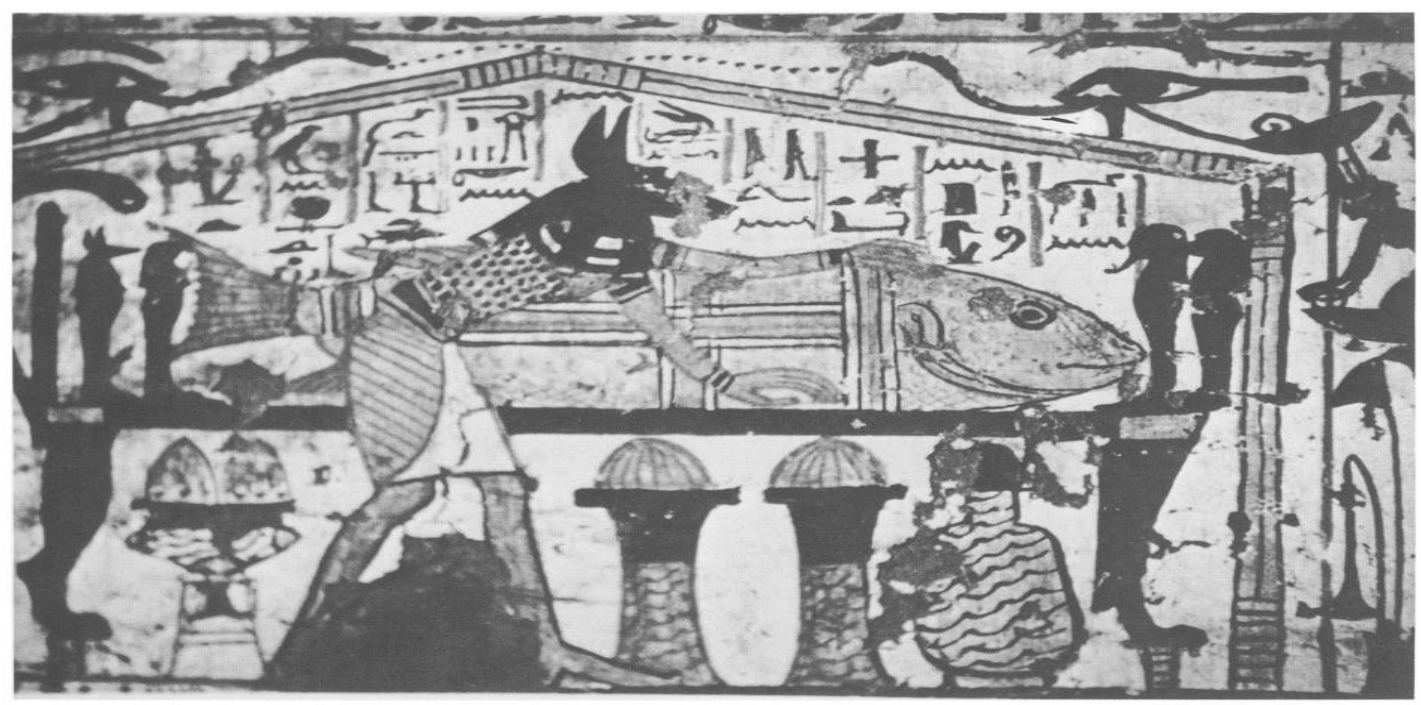

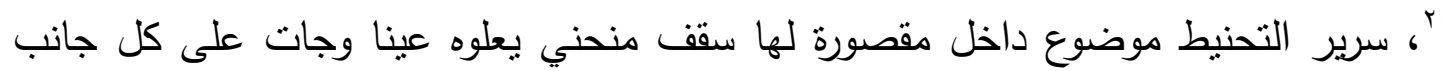

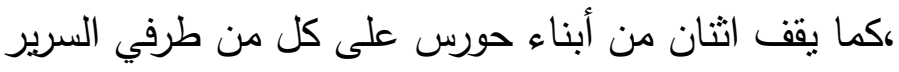




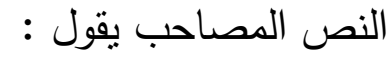

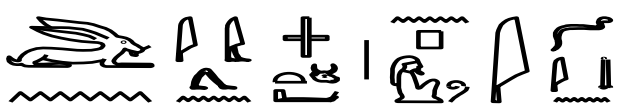

k n Dt AbDw Dd mdw in inpw imy xnt ii $n$ wn n(fr) m sAw "كلمات ثقال بواسطة انبو الذي في المقدمة ،حضر من اجل وننفر لحمايتك للأبد ، يا سمكة / ابجو يُلاحظ ان السرير هنا له أقدام اسد ولكن بدون رأس ويعلوه مقصورة أو تابوت برقد داخله الملك

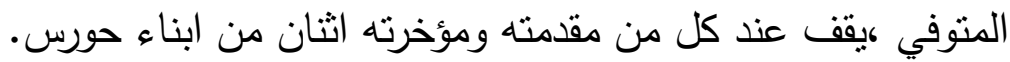

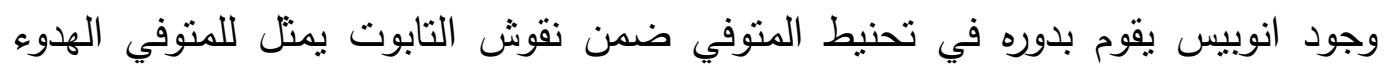

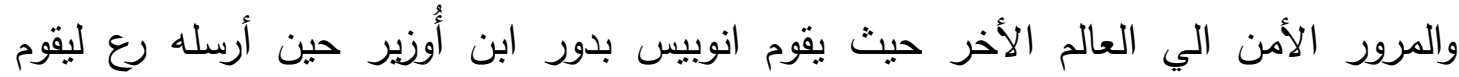

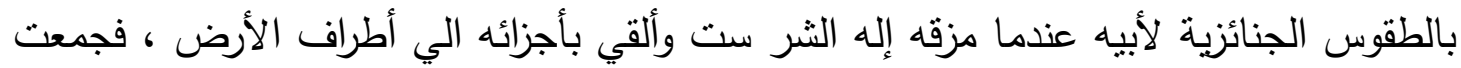

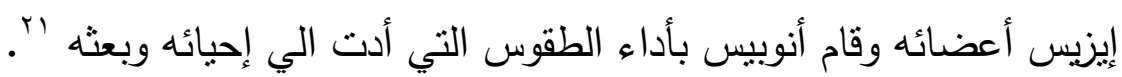

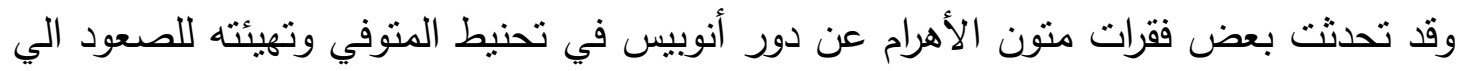
pyr. 1122c السماء فتنكر

$$
4 p-45 \text {. }
$$

أحشائه غسلت بواسطة انوبيس $\quad$ iai im Xtw .f in inpw ثم في فقرة 7-pyr796 وهي تتحدث عن صعود المتوفي الي السماء

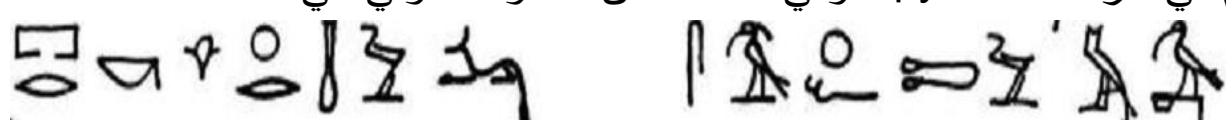

$\operatorname{Pr} k$ xr mdw inpw sAx. f sw $m$ tHwt أنت تصعد بصوت انوبيس ،هو الذي جعلك روح مثل تحوت

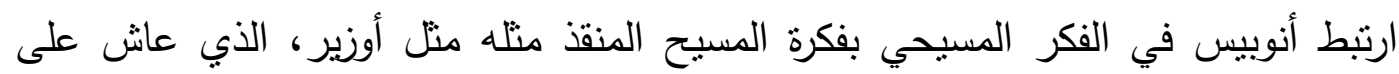

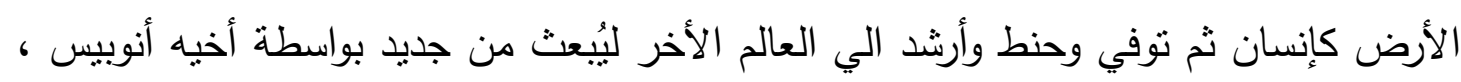

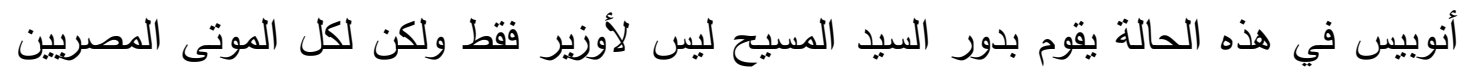

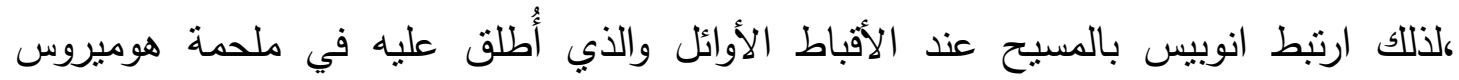

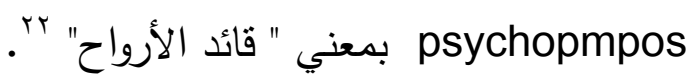
يري Dawson ان السرير على هيئة أسد لم يكن يستخدم حتي يتم الانتهاء من مراحل

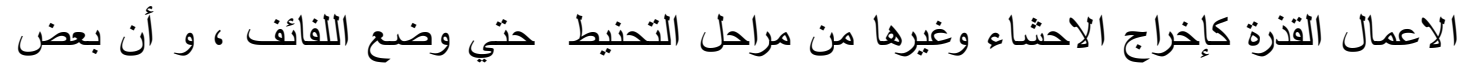

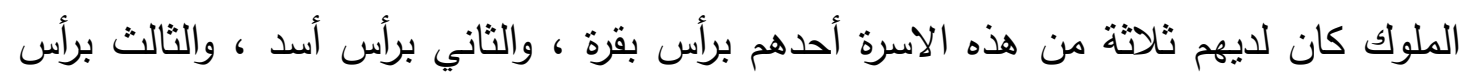


فرس نهر يتضح ذلك من المناظر في مقبرة سيتي الأول ، والبقايا التي عثر عليها في مقبرة

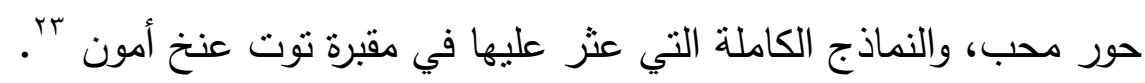

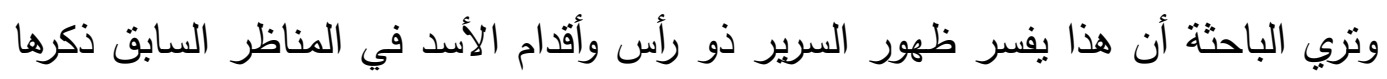

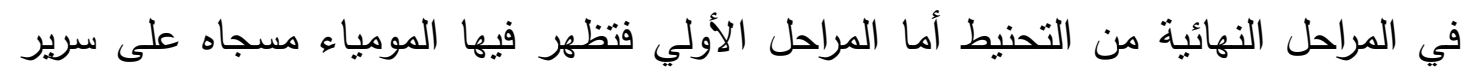

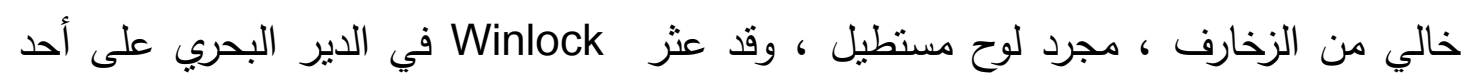

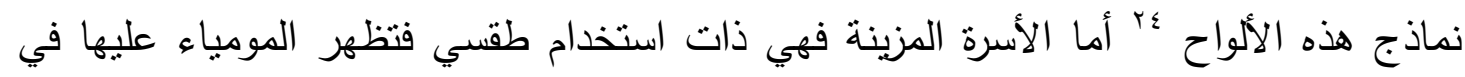

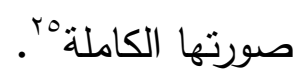

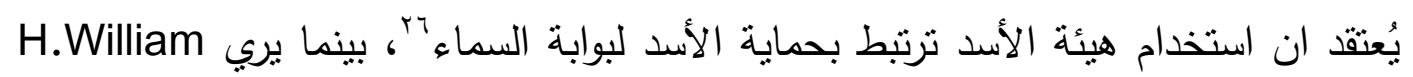

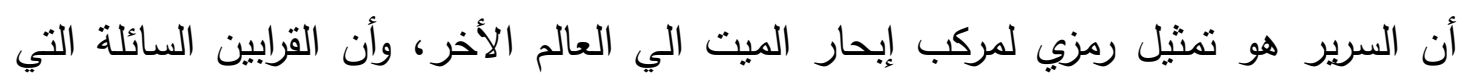

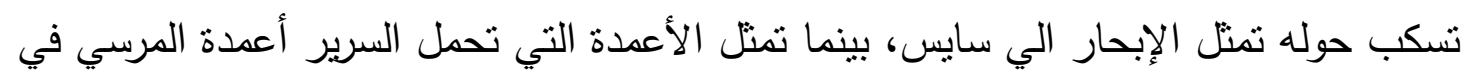

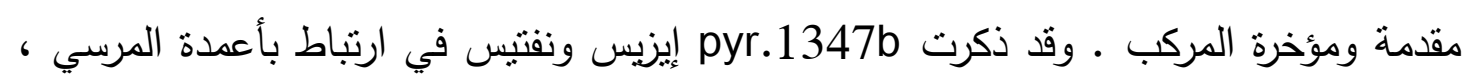

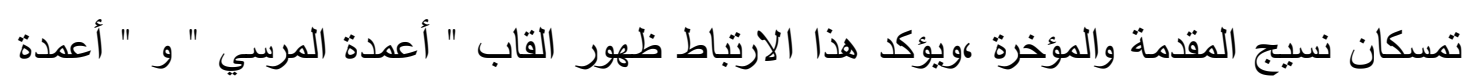
المرسي العظيمة " حملتها كاهنات معبد

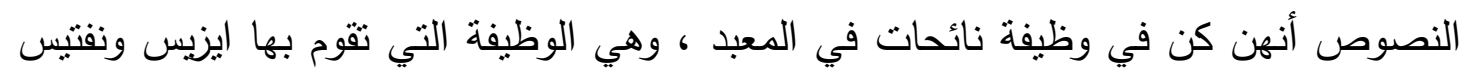

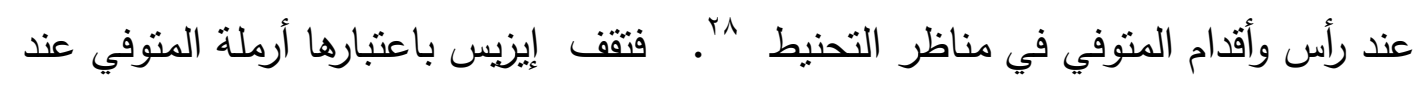

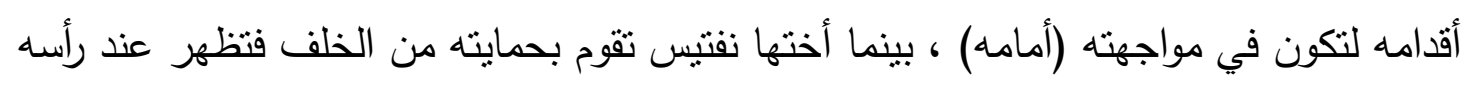

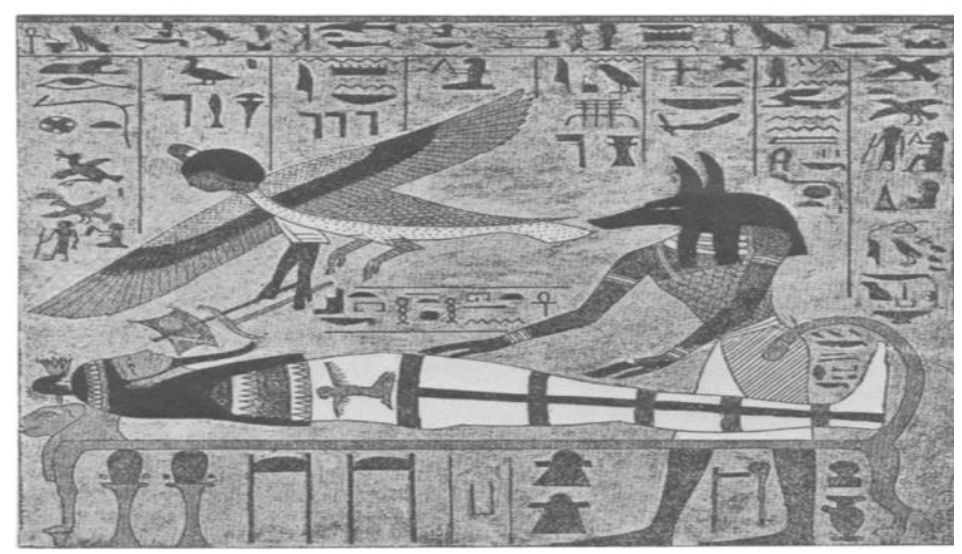

• وهو ما يفسر تحول المعبودتين فيما بعد الي معبودات كونية تمثل إيزيس الجنوب ونفتيس

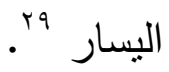


وفي نقوش مقبرة الوزير باسر ‘TT106(شكل TT) بينما يقوم أنوبيس بوضع يديه على المومياء

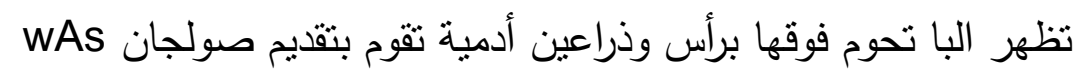

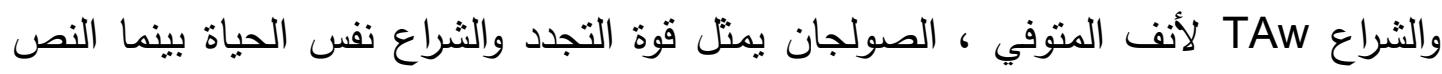

$$
\text { المصاحب يقول : }
$$

st k nt niwt nHh ht anx "r مكانك في مدينة الأبدية ---تعيش ل

بينما نظهر البا فوق السرير الأوزيري ضمن نقوش احد التوابيت بالمتحف المصري رقم

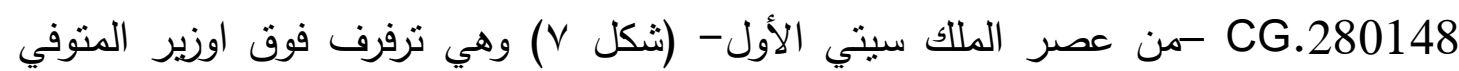

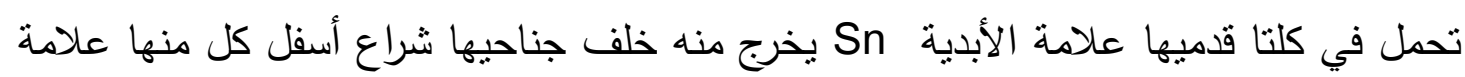
فDDm

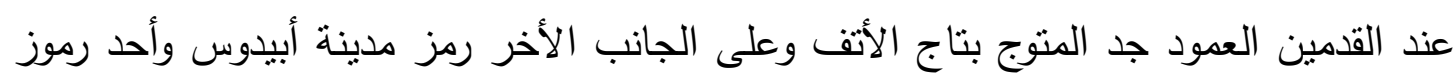

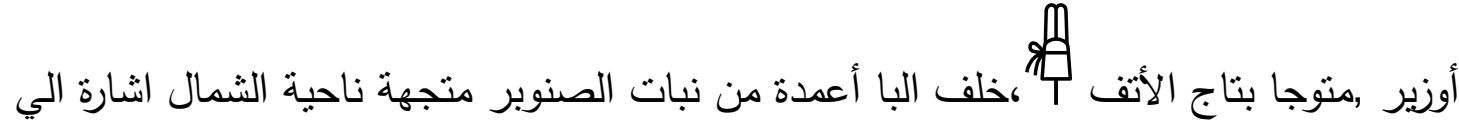

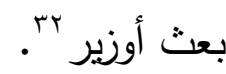

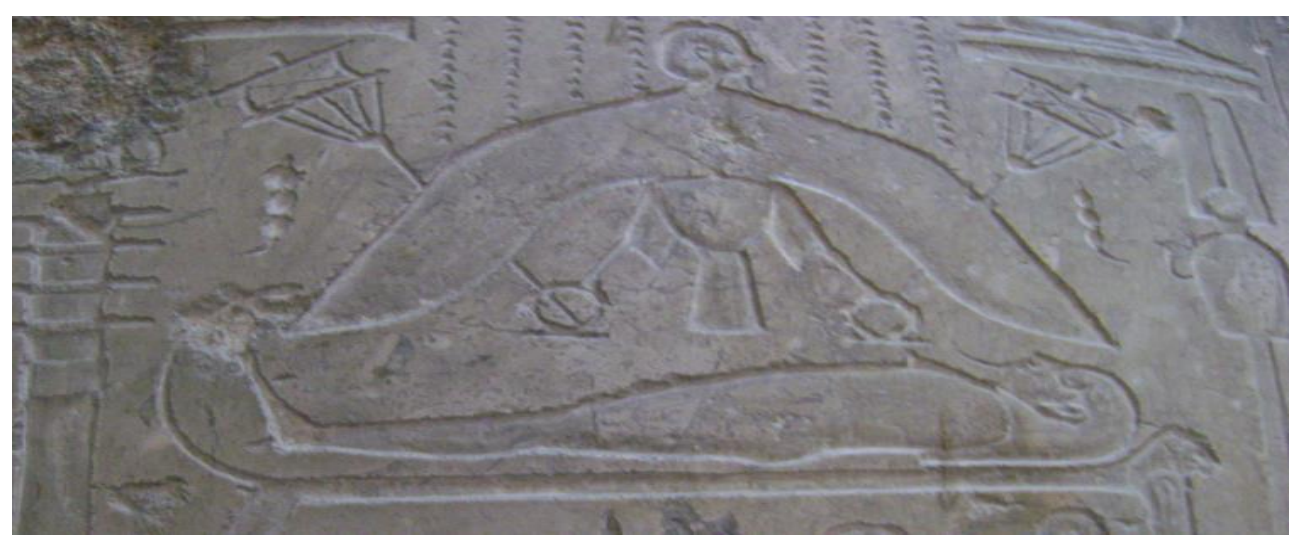

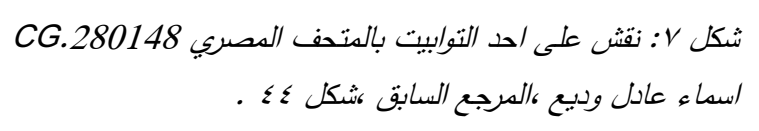

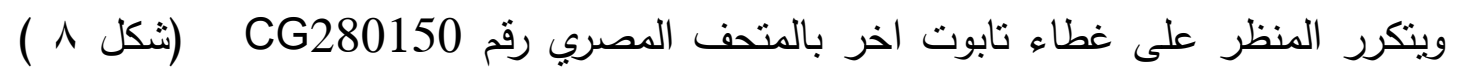

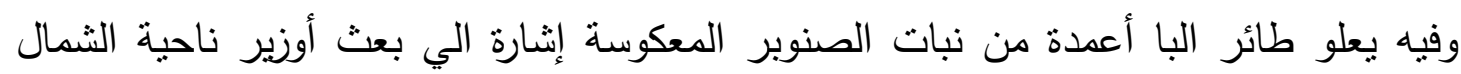

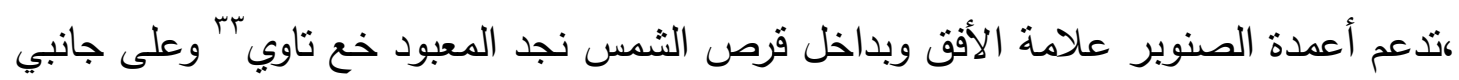


علامة الأفق عيني حورس الحاميتين ، بينما تقف نفتيس عند رأس سرير التحنيط وتقف إيزيس عند نهايته الأخري

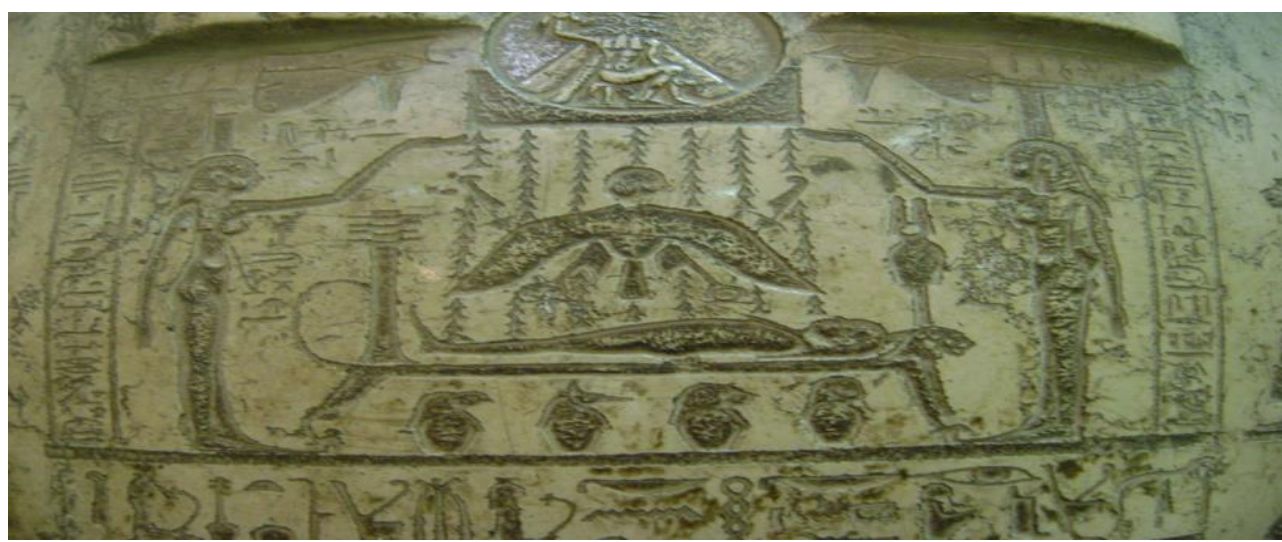

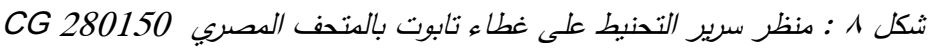

Lacau,P.,Sarcophages antérieures au Nouvel Empire,II , CG.28087-28126

,1906,pl.135.

مما سبق يتضح أن : بدأ ظهور سرير التحنيط ضمن المناظر الجنائزية منذ بداية الدولة الحديثة ، يتضح من تلك المناظر ان السرير المستخدم في بداية عملية التحنيط يكون مكون من ألواح مستقيمة خالية من الزخارف تتجه عليه مومياء المتوفي الي جهة اليمين بينما يستخدم السرير المزخرف بهيئة الأسد- أو بأكثر من هيئة كالبقرة أوفرس النهر في الآثاث الجنائزي الملكي - بعد انتهاء طقوس التحنيط، وتتجه المومياء عليه الي جهة اليسار في معظم الحالات وهو ما يتفق مع فكر المصري القديم منذ عصوره الأولي في ارتباط أرواح الموتي باتجاه الثمال حيث اتجاه النجم القطبي والنجوم التي لا تفني • 
' ' - على سبيل المثال :ضمن زخارف مقبرة تاوسرت رقم ع ا في وادي الملوك ،المنظر رقم 19 جانب غرفة :

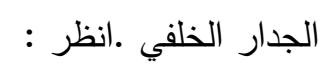

Lanzone, R. V., Dizionario di mitologia egizia (Amsterdam, 1974; reprint of Turin, I88I -4), p. 70.

$$
\text { - نقوش فيلة ،غرفة أوزير .أنظر : }
$$

. Berlin photos 1146. also Lanzone, op. cit. pls.265 261,

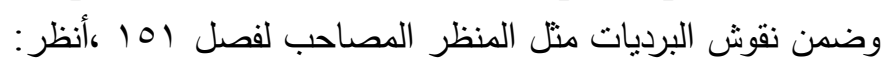

Budge, E. A. W., The Book of the Dead. III. The Papyrus of Ani (London, 1913), pl. 34 and Piankoff, A., Mythological Papyri (New York, 1957), pl. 22 (Papyrus of Djed-Khonsu-iuf-ankh II).

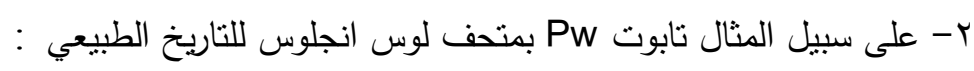

Jojn, J.A , Journey through Eternity: Egyptian Funeral Art, A Thesis presented to the faculty of California state University ,Master of Art.2000, p.11,21.

$$
\begin{aligned}
& \text { لوحة خشبية من مؤخرة تابوت بمتحف الفن الجميل بنيويورك ترجع الي القرن الأول الميلادي يظهر عليها }
\end{aligned}
$$

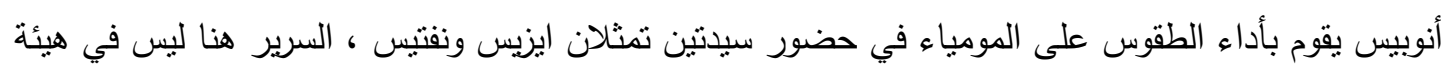

Museum of fine Art, Egyptian and Near Eastern art, Annual Report of the Museum of fine Art,vol.103,22.

3 -Vandijk, J., Essays on ancient Egyptian : in Honour of Herman Te Velde, Egyptologica Memoirs I(1997), Greningen,p.359.

$$
\text { يلفت Jandijk, J النظر الي العثور على نابوت على هيئة سرير تحنيط محفوظ بالمتحف الصصري برقم }
$$

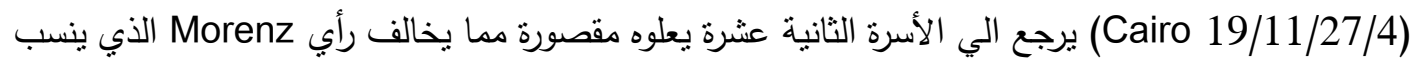

$$
\text { هذه النوعية الي العصر اليوناني الروماني }
$$

Morenz, Religion und Geschichte des Alten Ägypten Gesammelte Aufsätze,Cologne und Vienna,1975,5

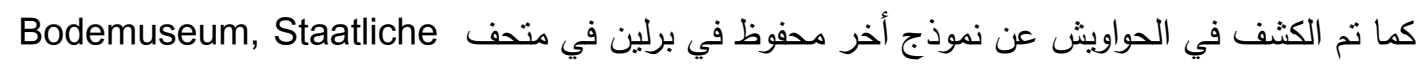

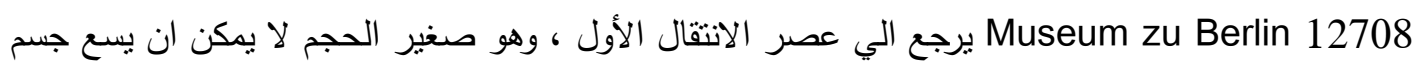

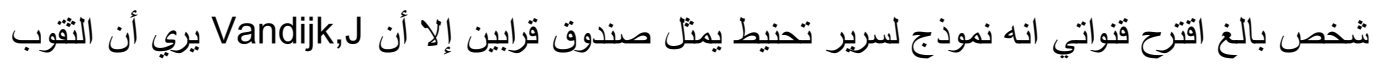

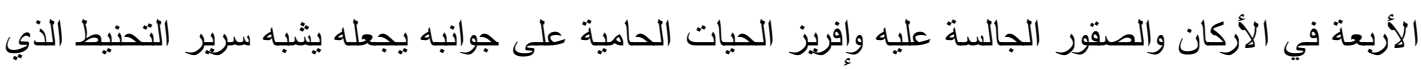
ظهر ضمن نقوش مقبرة شاشنق الثالث في تانيس الأنس

Kanawaty,G., The Rock Tombs of El-Hawawish,The Cemetry of Akhmim,VII , Sydney 1987,55, pl.15-16 
Montet,P., Les Constructions et Les Tombeau de Chéchanq III a Tanis,Paris 1960 ,67$69, \mathrm{pl} .30$.

4 - Jones,M.,Jones ,a.M., 1982), The Apis House project at Mit Rahinah first season ,1982,JARC 19 ,p.55.

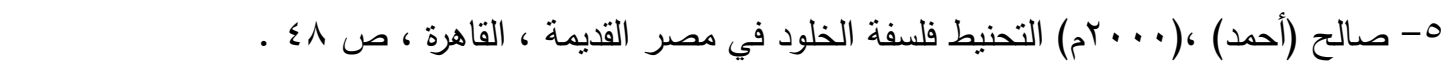

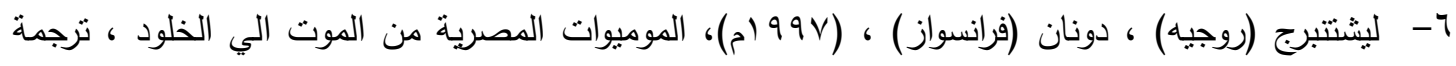

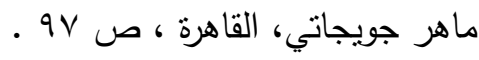

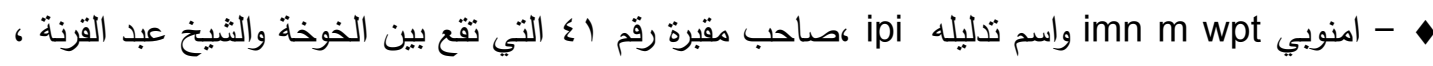

عاش في عصر حور محب و ستي الأول ، وقد حمل القاب الكاتب الملكي ومدير أملاك أمون ،زوجته هي

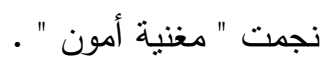

Assman ,J., Das Grab des Amenemope TT41 ,Theban III,Mainz19991.

7 - Ikram,S., López,Grande,M.J.,Three Embalming Caches from Dra Abu el Naga ,BIFAO 111(2011),pp.205-228.

8 - Dawson,W.R.,Making a Mummy ,JEA 13( 1927 ),p.46f,pl.XVIII.

9 - Goldstein,S.M.,Egyptian And Near Eastern Art, Bulletin(St. Louis Art Museum ) New Series vol.19No.4(1990)p.25,29.

10 -Schmitz,B.,Sarg des Djed- Bast-iu-ef-ankh,in:A.Eggebrecht,ed., Stuche nach Unsterblichkeit Hildesheim and Mainz,1990,p.28-29; Schulz,R.,A Corn Mummy decoded ,The Journal of the Walters Art museum 63(2005), fig.1.

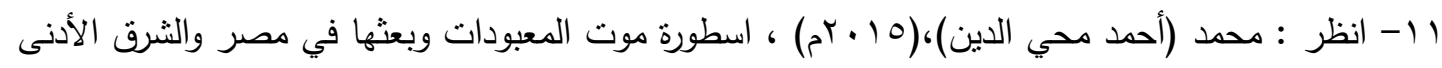
القديم (دراسة دينية مقارنة) ، رسالة ماجستير غير منشورة ، كلية الآداب ،جامعة المنيا.

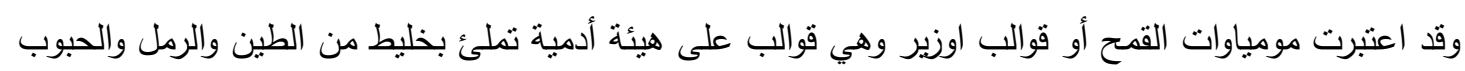
وينم لفها باللفائف الكتانية وريها بالماء حتي تنبت تأكيدا لفكرة الإحياء .

Schulz,R.A., A Corn Mummy decoded ,The Journal of the Walters Art Museum ,vol.63(2005) p.5,fig.1

r إ- نرجع أقدم أدلة اثرية على استخدام الأواني الكانوبية الي أواخر الأسرة الرابعة حيث عثر عليها قريبة من

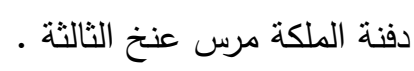

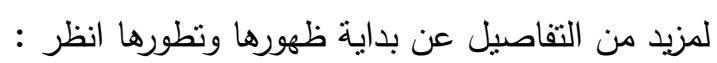

Dodson,A., Canopic Equipment of the Kings of Egypt ,Routledge 2009

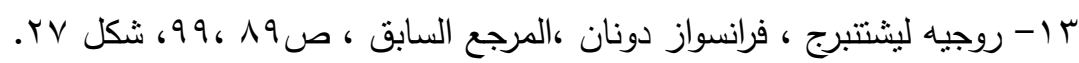

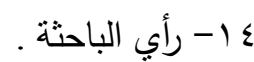

15 - Revan,M.J.,Egyptian Concepts on the Orientation of the Human body ,JEA.91 (2005),p.48.

1.

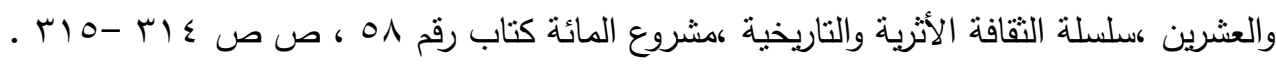

iv - Vandijk,J., op.cit., p.359. 
18 - Assman,Das Grab der Mutiridis,AVDAIK 13(1977),74.Wiatkus,W., Zur Deutung einiger a Poträischer Gotter in den Gräbern im tal der Königinnen und im Grab Ramsses Iii, GM.99(1987),pp.68-70.

- خع بخنت : رسام حمل لقب خادم في قاعة العدالة مما يشير اليي أنه عمل تزيين مقابرالملوك ، عاش

في دير المدينة في عهز رمسيس الثاني ، وهو ابن سننجم •

• - ارتبطت سمكة AbDw في الأساطير المصرية بمركب الثمس فهي تصحبها ووظيفتها الإبلاغ عن

اقتراب الثعبان عدو إله الثمس ، تثابه اسمها مع اسم مدينة أبيدوس ربط بينها وبين أوزير وكان سبب تقديس لقديسها

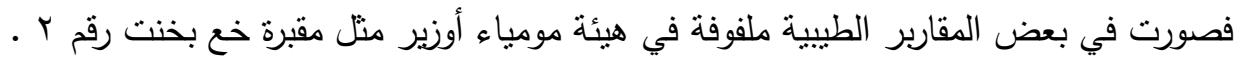

Bonnet,H., Abdufisch, Reallexikon der agyptischen Religionsgesschichte, New York 2000, s.193-194.

${ }^{20}$-Ritner, R.K., Anubis and the Lunar disk ,JEA71 (1985) ,p.152,pl.XV.

21 - Goldstein,S.M., op.cit., p.28,29.

22 - Carus, P., The Cpnception of the Soul and the Belief in Resurrection,p.414

23 - Dawson, W.R., Making a Mummy, JEA 13( ) p.42.

24 -Winlock, H.E., Excavations at Thebes, BMMA 17(1922)pl.II,p.34

25 -Jones, M; Jones, A.M., The Apis house project, p.54.; Dawson, W.R., Making a mummy,p.42.

A Thesis presented to ${ }^{26}$-John,J.A.,Journey through Eternity :Egyptian Funeral Art, the Faculty of California state University,Master of Art 2010 ,p.21.

"البيث العظيم " وهو اسم مقصورة المعبودة نخبت المعبودة الرئيسية لمدينة نخن - الكاب، الكوم

$$
\text { الأحمر حاليا على الضفة الغربية للنيل- وحامية التاج الأبيض }
$$

Fakhry,A., The monuments of Senferu at Dahshur : The Vally Temple ,Cairo 1961, p.73.

28 -Willems,H., The Coffin of Heqata ,Cairo JdE.36418,Leuven1996,p.117.

29 - Revan,M.J.,Egyptian Concepts on the Orientation of the Human body ,JEA.91 (2005),p.48.

• ץ- باسر : احد وزراء عصر رمسيس الثاني وسيتي الأول ، حمل لقب الكاهن الأول لأمون ، مقبرته رقم

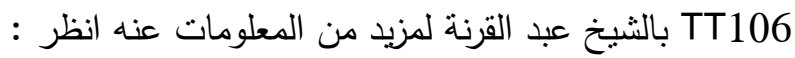

Kitchen K.A., Rammeside Inscriptions, Translated \& Annotated,Translaions, III,1,Oxford 1980 ,pp.1-36.

31- Carus,P., The Cpnception of the Soul and the Belief in Resurrection,p.413

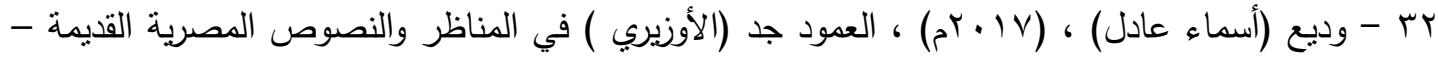

دراسة فنية حضارية-رسالة ماجستير غير منشورة ،جامعة المنيا ،كلية الآداب ،ص عـ، شكل عـ . .

rr- خع تاوي : عرف في نصوص الأهرام 518 Pyr 242,423 بأنه ابن الآله رع للبلدان الأجنبية ، وكان عندان

أمراء جبيل يزينون برسومه اسلحتهم وحليهم باعتباره رع البلدان الأجنبية .

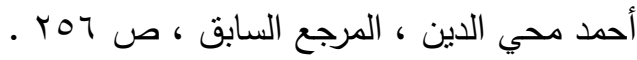

